\title{
Virtual Screening of Combinatorial Libraries across a Gene Family: in Search of Inhibitors of Giardia lamblia Guanine Phosphoribosyltransferase
}

\author{
ALEX M. ARONOV $\uparrow$ NARSIMHA R. MUNAGALA, IRWIN D. KUNTZ, AND CHING C. WANG* \\ Department of Pharmaceutical Chemistry, University of California, San Francisco, California 94143-0446
}

Received 13 February 2001/Returned for modification 2 April 2001/Accepted 18 June 2001

\begin{abstract}
Parasitic protozoa lack the ability to synthesize purine nucleotides de novo, relying instead on purine salvage enzymes for their survival. Guanine phosphoribosyltransferase (GPRT) from the protozoan parasite Giardia lamblia is a potential target for rational antiparasitic drug design, based on the experimental evidence, which indicates the lack of interconversion between adenine and guanine nucleotide pools. The present study is a continuation of our efforts to use three-dimensional structures of parasitic phosphoribosyltransferases (PRTs) to design novel antiparasitic agents. Two micromolar phthalimide-based GPRT inhibitors were identified by screening the in-house phthalimide library. A combination of structure-based scaffold selection using virtual library screening across the PRT gene family and solid phase library synthesis led to identification of smaller (molecular weight, <300) ligands with moderate to low specificity for GPRT; the best inhibitors, GP3 and GP5, had $K_{i}$ values in the 23 to $25 \mu \mathrm{M}$ range. These results represent significant progress toward the goal of designing potent inhibitors of purine salvage in Giardia parasites. As a second step in this process, altering the phthalimide moiety to optimize interactions in the guanine-binding pocket of GPRT is expected to lead to compounds with promising activity against $G$. lamblia PRT.
\end{abstract}

Computer-aided drug design in combination with combinatorial chemistry approaches, whereby focused or diverse combinatorial libraries can be designed using computational methods, is becoming increasingly important in the process of drug discovery for parasitic targets $(7,11)$. A number of groups have reported on the successful design of inhibitors directed against trypanosomal (2, 4, 15-16), leishmanial (6), malarial (19), and tritrichomonal $(3,27)$ targets active in the $10 \mathrm{nM}$ to $50 \mu \mathrm{M}$ range. However, with the number of compounds that could be generated by combinatorial chemistry growing exponentially, it has become apparent that chemical diversity has surpassed the capacity of high-throughput screening. In the case of antiparasitics research, which is concentrated in a limited number of mostly academic labs, the need for more rapid ligand screening tools has become apparent. Recently, in silico methods for database screening have come to the forefront of drug discovery (30). By accelerating the screening process, these methods are able to capitalize on the potential of virtual combinatorial libraries. While a number of recent reports have focused on structure-based pruning of the virtual combinatorial libraries built around a given preselected scaffold, there has been a growing trend toward combinatorial scaffold evaluation against a number of biological targets. Evaluation of binding preferences for combinatorial libraries across a range of targets could, in principle, provide information about scaffold generality or selectivity as related to the target selection (M. L.

\footnotetext{
* Corresponding author. Mailing address: Department of Pharmaceutical Chemistry, 513 Parnassus Ave., Box 0446, University of California, San Francisco, CA 94143-0446. Phone: (415) 476-1321. Fax: (415) 476-3382. E-mail: ccwang@cgl.ucsf.edu.

$\dagger$ Present address: Vertex Pharmaceuticals Inc., Cambridge, MA 02139 .
}

Lamb, K. W. Burdick, S. Toba, M. M. Young, A. G. Skillman, X. Zou, J. R. Arnold, and I. D. Kuntz, unpublished data.).

All protozoan parasites lack the ability to synthesize purine nucleotides de novo. Instead, they utilize purine salvage pathways to convert the host organism's purine bases and nucleosides to the corresponding nucleotides (31). Purine phosphoribosyltransferases (PRTs) catalyze the $\mathrm{Mg}^{2+}$-dependent synthesis of purine nucleotides via reaction of a purine base with $\alpha$-D-5-phosphoribosyl-1-pyrophosphate (PRPP). Crystal structures of the type I PRTs share a common Rossman's fold and a hood that is composed primarily of antiparallel $\beta$-sheets positioned around the enzyme's active site $(8,12,20-23,28)$. Inhibitors of PRTs that are able to block purine salvage in vivo could represent an efficient approach to antiparasite chemotherapy $(31,32)$.

Giardia lamblia, an anaerobic binucleate flagellated protozoan that causes intestinal infection, a condition termed giardiasis in mammals (1), relies primarily on two independent pathways for its nucleotide synthesis. Adenine PRT and guanine PRT (GPRT) catalyze the synthesis of AMP and GMP, respectively. Due to a lack of interconversion between the two purine nucleotide pools, either of the two enzymes could serve as a potential target for giardiasis chemotherapy (33).

G. lamblia GPRT shows little homology with the known sequences of other purine PRTs (26). It possesses a rather unique guanine-only specificity, while exhibiting very low activity with hypoxanthine as a substrate. A recently published high-resolution X-ray structure of G. lamblia GPRT (23) demonstrated a number of structural differences between GPRT and other known PRTs. The purine is stacked between two aromatic residues, Trp180 and Tyr127. While a Trp residue has been also seen at this first position in Toxoplasma gondii hypoxanthine-guanine-xanthine PRT (HGXPRT), tyrosine and phenylalanine are present at the corresponding position in 


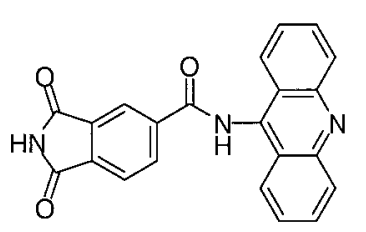

$\mathrm{IC}_{50}=40 \mu \mathrm{M}$

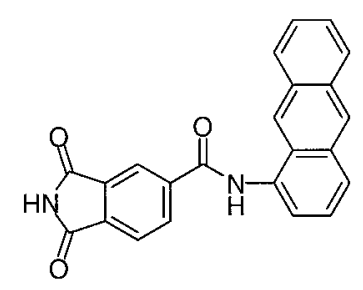

$\mathrm{IC}_{50}=48 \mu \mathrm{M}$
FIG. 1. G. lamblia GPRT inhibitors identified through screening of the in-house chemical database.

Tritrichomonas foetus HGXPRT and human hypoxanthineguanine PRT (HGPRT), respectively. The unusual substitution is observed at the bottom of the purine binding site, with Tyr127 taking the place of the typically well-conserved Ile or Leu residue. Another structural difference can be noted in the position of the conserved Lys residue, which has been shown to interact with exocyclic $\mathrm{O} 6$ of the purine in all of the known structures of purine PRTs. Lys152 of GPRT positions its $\varepsilon-\mathrm{NH}_{2}$ group $6.3 \AA$ away from the $\mathrm{O} 6$ of guanine, in sharp contrast to the typically observed distance of $3 \AA$, with two ordered water molecules spanning the distance. Despite the noted structural differences, the active site preserves the bifocal character observed in other purine PRTs, formed by the stacking interaction at the purine binding site and the hydrogen bonding interactions at the $5^{\prime}$-phosphate binding site of PRTs.

Having previously reported on the successful design of selective $T$. foetus HGXPRT inhibitors based on the phthalimide scaffold (3), we looked into the possibility of designing $G$. lamblia-specific GPRT inhibitors. Two bulky phthalimidocarboxanilides (Fig. 1) were identified as the most active leads. At this point we decided on the following two-stage strategy in our quest for GPRT ligands. The first stage would involve using the virtual and synthesized phthalimide libraries to focus the search on smaller substituents that would bind to the 5'-phosphate binding site. Following identification of such moieties, we could then move to design novel scaffolds that would be better tailored for the GPRT purine binding site than our starting phthalimides. As part of this project, we will describe the design of small phthalimide-containing molecules that retain inhibitory activity against GPRT. Since a decrease in human HGPRT activity has been demonstrated to be responsible for gouty arthritis and the Lesch-Nyhan syndrome, the compounds being developed as G. lamblia GPRT inhibitors should not interfere with mammalian HGPRT activity. Herein we report on the utility of virtual combinatorial library screening for the discovery of micromolar inhibitors of G. lamblia GPRT and on applications of virtual library screening across the representatives of the target gene family for analyzing species selectivity in the process of scaffold selection.

\section{MATERIALS AND METHODS}

Materials. Libraries of phthalimide-containing GPRT inhibitors were synthesized, purified, and analyzed as reported previously (3). Guanine and the tetrasodium salt of PRPP were purchased from Sigma Chemical Co. (St. Louis, Mo.) and are of the highest purity available.
Enzyme assays. Isolation $(5,14)$ and assays (34) of recombinant $G$. lamblia GPRT and human HGPRT from transformed Escherichia coli were performed as described previously. The substrates were present at the following concentrations: $20 \mu \mathrm{M}$ guanine $\left(K_{M}=16.4 \mu \mathrm{M}\right)$ and $1 \mathrm{mM}$ PRPP $\left(K_{M}=25.6 \mu \mathrm{M}\right)$. The compounds tested were dissolved in dimethyl sulfoxide- $d_{6}$, and concentrations were determined by integration of nuclear magnetic resonance peaks with methylene chloride as an internal standard. The concentration of dimethyl sulfoxide in the assays was kept at $10 \%$.

Structure analysis. The following crystal structures were used in the virtual library screening comparison: T. foetus HGXPRT (1hgx) as determined by Somoza et al. (28), G. lamblia GPRT (1dqn) by Shi et al. (23), T. gondii HGXPRT (1qk3) by Heroux et al. (13), Trypanosoma cruzi HGPRT (1tc1) by Focia et al. (10), E. coli HGPRT (1a95) by Vos et al. (29), and human HGPRT (1hmp) by Eads et al. (8). Positions of the purine/pseudopurine ligands (e.g., guanine, immucilin $\mathrm{G}$ phosphate, formycin B, etc.) were used to align the structures. The Sybyl (version 6.5; Tripos Associates, St. Louis, Mo.) software package was used for display and analysis of the structures and to compute all atom Amber charges for the proteins.

Virtual library construction. UC_Select (25) in combination with the Daylight version of the Available Chemicals Directory was used to identify original reagent sets, as well as for the elimination of reagents that had unattractive chemical or pharmaceutical properties. Similarity and superstructure searches of the Available Chemicals Directory were performed with Daylight's Merlin system (version 4.61; Daylight Chemical Information Systems, Inc., Santa Fe, N.M.), using a Tanimoto similarity metric and Daylight's hashed connectivity fingerprints. Sybyl's CONCORD module was used to build the virtual reagent library. The virtual library was prepared within Sybyl using in-house SPL scripts, and Gasteiger-Marsili charges were computed.

Docking. DOCK4.01 (9) was used to screen the virtual phthalimide libraries against the target PRTs. In every case, the previously described (3) three-step docking procedure was used involving (i) library preorientation, followed by (ii) rigid docking and (iii) flexible scoring. Spheres were chosen by superimposing phthalimide on the purine or pseudopurine atom positions derived from the respective crystal structures as reported previously (3). The docking parameters were held constant for runs across the PRT gene family and will be available upon request.

\section{RESULTS AND DISCUSSION}

Choice of scaffold and chemistry. Having previously reported on the successful design of selective submicromolar $T$. foetus HGXPRT inhibitors based on the phthalimide scaffold, we looked into the possibility of designing inhibitors that would target a related enzyme, G. lamblia GPRT. Due to the specificity difference between the $T$. foetus and G. lamblia PRTs, phthalimide would not be generally expected to serve as an efficient scaffold in the context of the GPRT binding site. The imide portion of the molecule mimics the $\mathrm{C}_{6}-\mathrm{N}_{1}-\mathrm{C}_{2}$ of xanthine, one of the natural substrates of HGXPRT. It could be expected to form unfavorable interactions with the backbone carbonyls of Asp181 and Asp187, which directly interact with the exocyclic $\mathrm{N}_{2}$ of guanine. However, due to the general similarity in the shape of the active sites between the $T$. foetus and $G$. lamblia PRTs, we decided that screening of our inhouse phthalimidocarboxanilide database for inhibitory activity against GPRT could potentially result in identification of a starting point for our lead discovery efforts. This approach resulted in identification of two active molecules (Fig. 1). Both phthalimide derivatives contained bulky fused ring systems that most likely derived much of their binding energy from burying the large hydrophobic substituents. We set out to design smaller, less lipophilic molecules that would retain their activity against GPRT.

Virtual library construction and evaluation. As the first step, UC_Select (25) was used to perform initial database mining. Lipinski "druglikeness" criteria (17) served as a filter, followed by visual inspection and removal of unreactive mol- 


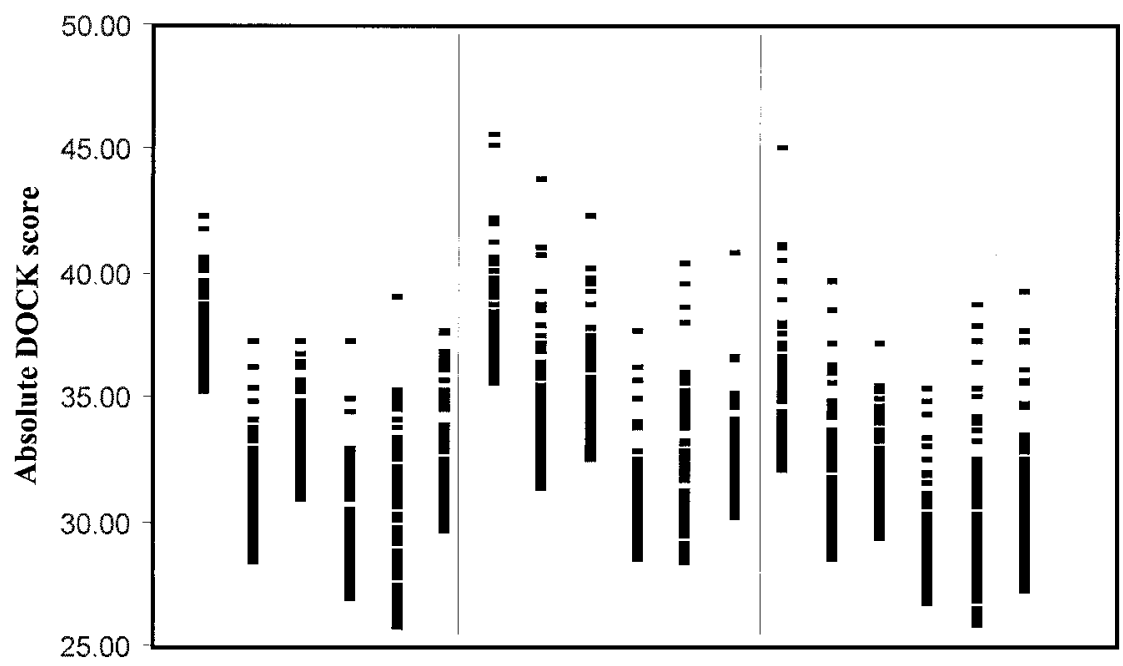

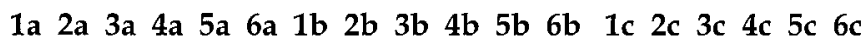

FIG. 2. Comparative performance of phthalimide-based virtual libraries across the PRT gene family. 1, T. foetus HGXPRT; 2, G. lamblia GPRT; 3, T. gondii HGXPRT; 4, T. cruzi HGPRT; 5, E. coli HGPRT; and 6, human HGPRT. The following letters refer to libraries: a, phthalimidocarboxanilides; $\mathrm{b}$, primary phthalimidocarboxamides; c, secondary phthalimidocarboxamides. The $y$ axis represents absolute DOCK score values for the top 100 scoring members of individual libraries. Actual scores were negative in all cases.

ecules, to produce three separate reagent sets: 599 anilines, 460 primary amines, and 298 secondary amines (for a more detailed description of the procedure, see reference 3). Virtual phthalimide libraries were prepared as described in Materials and Methods. The anilide, primary amide, and secondary amide libraries were treated independently.

Six publicly accessible crystal structures of purine PRTs were used to put together a target library. All of the structures chosen have the trademark PRT flexible loop in the "open" conformation or disordered (in the case of $T$. foetus HGX PRT). This loop has been shown to act as a flap that covers the catalytic pocket to shield the oxocarbonium transition state from nucleophilic attack by bulk solvent (21-22). As a control, two purine PRT structures with the loop in the "closed" conformation were also chosen for docking: human HGPRT (1bzy) (24) and Plasmodium falciparum HGXPRT (1cjb) (22). In the case of the control structures we observed that $>98 \%$ of the library members received a positive score, i.e., were classified as nonbinders (data not shown). However, that would be inconsistent with our previously published results (3) showing that human HGPRT was inhibited by most phthalimides in the 25 to $250 \mu \mathrm{M}$ range. This result suggests that the inhibitors bind to the open or partially open form of the enzyme.

The three virtual libraries were successively docked to all of the target structures. The results for 100 top-scoring compounds from each run are shown in Fig. 2. We have previously speculated (3) that the phthalimide scaffold could potentially have built-in selectivity for $T$. foetus HGXPRT over human HGPRT, since the human enzyme does not act on xanthine. Indeed, based on our virtual screening data, the tritrichomonal enzyme has the highest level of susceptibility to inhibition by phthalimides, with $T$. gondii HGXPRT as the second highest on our PRT list. Of the three HGPRTs on the list, the human enzyme appears to be the most susceptible, while the trypanosomal isozyme would be predicted to bind poorly to the com- pounds in the three libraries. Based on the virtual performance of the phthalimides against the two HGXPRT enzymes in the panel, one would also anticipate a similar showing against the malarial enzyme. Given the kinetic parameters for $P$. falciparum HGXPRT (29 $\mu \mathrm{M}$ for xanthine) (37), phthalimides represent a good potential scaffold for designing inhibitors of the malarial enzyme. In conclusion, the variation in the library performance across the targets could be deconvoluted into (i) scaffold preference based on substrate specificity for the purine binding site and (ii) structural differences within the $5^{\prime}$-phosphate binding pocket.

Evaluation of consensus substituents. For G. lamblia GPRT, the primary amide library would be predicted to be the most active of the three virtual libraries tested. In all three cases, based on the results of virtual library screening, building selectivity into the system would be expected to be one of the major challenges. As the first step in our drug design effort against G. lamblia GPRT (see the introduction), the top 10\% of the scorers in both the anilide and primary amide libraries were visually evaluated. A number of consensus substitutions shared by most of the top scorers were identified, and the best ligands were chosen for synthesis. In the case of the anilide library, a small substituent at the meta position in the aromatic ring was predicted to be beneficial; most top scorers had a methyl or a chloro substituent placed at that position. A $p$ fluoro group was also found to be prevalent among the top scoring anilides. A number of different substituents were placed at the ortho position, occupying a region proximal to the flexible loop. The $o$-chloro group was chosen as representative of that class of compounds. In the primary amide library, a consensus substituent was a para-substituted phenethyl group, with small substituents predicted to be best at this position.

A straightforward chemical scheme implemented to prepare the consensus substituted phthalimides (Fig. 3) has been described previously. Briefly, we used trimellitic anhydride as a 
<smiles>O=C(O)c1ccc2c(c1)C(=O)OC2=O</smiles><smiles>CCCC</smiles>

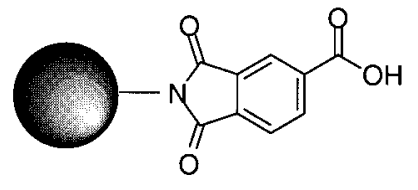<smiles>O=C(N[I+]I)c1ccc2c(c1)C(=O)NC2=O</smiles>

inhibitor library

FIG 3. Chemical scheme for preparation of the consensus substituted phthalimides. a, $\left(\mathrm{NH}_{4}\right)_{2} \mathrm{CO}_{3}$, melt; b, trityl chloride resin, DIPEA, $\mathrm{CH}{ }_{3} \mathrm{CN}$; c, amine library, PyBrOP, DIPEA, DMA, 5 days; d, TFA, $3 \mathrm{~h}$. The sphere represents resin support.

starting material, which upon conversion to 4-carboxyphthalimide was attached to the trityl chloride-functionalized resin. Target compounds were produced on solid support and were cleaved from the resin in the final step. Six compounds bearing consensus substitutions were prepared, and the results are shown in Table 1. Four of six compounds prepared showed GPRT inhibitory activity comparable to that of the lead compounds 1 and 2. GP5 was one of the most active and also the smallest anilide prepared. According to our model, it forms a hydrogen bond with Asp125 of GPRT. As predicted, a small hydrophobic substituent at the para position in the phenethyl group was well tolerated in the primary amide series, with GP3 being fivefold more active than the unsubstituted $N$-phenethyl amide (A. M. Aronov and C. C. Wang, unpublished data). Both GP3 and GP5 were shown to act as competitive inhibitors of GPRT, with $K_{i}$ values of 23 and $25 \mu \mathrm{M}$, respectively (data not shown).

TABLE 1. Inhibition of G. lamblia GPRT by phthalimides bearing consensus substitutions from the primary library

Ged ID ${ }_{\mathrm{GP}}^{a}$

${ }^{a}$ Compound identification.

${ }^{b} \mathrm{R}$, substitution of phthalimide.

${ }^{c} \mathrm{IC}_{50}, 50 \%$ inhibitory concentration.

${ }^{d}$ Remaining enzyme activity $(\%)$ at the stated inhibitor concentration.
The model for the binding of GP3 and GP5 within the active site of G. lamblia GPRT is shown in Fig. 4. We decided to prepare a number of analogues to test this binding hypothesis (Table 2). According to the model, placing an $o$-amino substituent instead of the $m$-hydroxyl is not expected to significantly alter activity, while small hydrophobic substitutions at the ortho position were disallowed due to unfavorable interactions with Asp125 and the backbone carbonyl of Tyr127. Indeed, the amino-substituted analog GP11 was only moderately less active than GP5, while addition of an $o$-methyl group in GP13 had a deleterious effect on activity. In the primary amide analog series, the model predicted that a larger hydrophobic substituent was somewhat disfavored at the para position, while a group containing two or more heavy atoms could not be accommodated within the $5^{\prime}$-phosphate binding pocket of GPRT, clashing with $\mathrm{C} \beta$ of Asp125 and C $\gamma$ of Ile134. A bromine introduced at the para position in GP9 faired worse than the chloro substitution in GP3, while GP10 did not possess any appreciable GPRT inhibitory activity. Placing small hydrophobic substituents at the meta position did not yield any improved binders. The most active compounds from both series showed little preference for G. lamblia GPRT when tested for inhibition of the human HGPRT (Table 3), with measured selectivity ranging from 1.6 -fold to $>7$-fold.

Overall, the experimental findings agree with the proposed model. The most apparent feature of the predicted binding modes for the two inhibitor series is that in both cases the aromatic ring positions itself in the $5^{\prime}$-phosphate binding site,

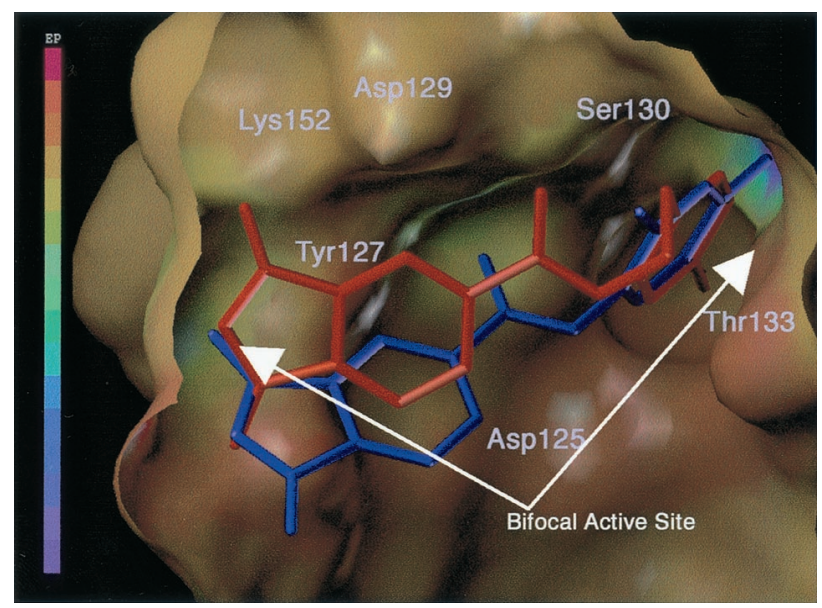

FIG. 4. Modeled binding mode of GP3 (red) and GP5 (blue) within the active site of G. lamblia GPRT (23). Electrostatic potential surface shown for GPRT (negative potential in red, positive in blue) was generated using MOLCAD (Sybyl, version 6.5; Tripos Associates). 
TABLE 2. Inhibition of G. lamblia GPRT $\left(\mathrm{IC}_{50}\right)$ by analogs of GP3 and GP5 from the secondary library

$\underset{\mathrm{Cmpd} \mathrm{ID}^{a}}{\mathrm{GP} 7}$

GP8<smiles>CCCc1cccc(Cl)c1</smiles>

GP9<smiles>CCCc1ccc(Br)cc1</smiles>

GP10<smiles>CCCc1ccc(OC)cc1</smiles>

GP11<smiles>Cc1ccccc1N</smiles>

GP12<smiles>CCOc1ccc(C)cc1C</smiles>

GP13

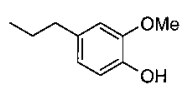

72

GP14

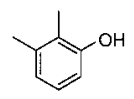

$>100$ (27)

${ }^{a}$ Compound identification.

${ }^{b} \mathrm{R}$, substitution of phthalimide.

${ }^{c}$ Remaining enzyme activity $(\%)$ at the stated inhibitor concentration is shown in parentheses. $\mathrm{IC}_{50}, 50 \%$ inhibitory concentration.

proximal to the conserved Ser130 and Thr133, while the phthalimide does not appear to have a single binding mode. The phthalimide scaffold is not optimal for the purine binding site, with the best compounds reported herein being approximately 1- to 2-orders-of-magnitude-weaker binders than the best rationally designed phthalimide-based $T$. foetus HGXPRT inhibitors described previously. These compounds show only low to moderate specificity for G. lamblia GPRT. However, this scaffold can be used in the initial stage of the design of GPRT inhibitors, serving as a vehicle to rapidly optimize the $\mathrm{N}$-amide portion of the inhibitors. The second part of our GPRT inhibitor design strategy is going to involve using the optimized $\mathrm{N}$-amide moieties of GP3 and GP5 to go back and design a
TABLE 3. Selective inhibition of $G$. lamblia GPRT by substituted phthalimides

\begin{tabular}{lcc}
\hline & \multicolumn{2}{c}{$\mathrm{IC}_{50}(\mu \mathrm{M})$ for $^{b}:$} \\
\cline { 2 - 3 } Cmpd ID $^{a}$ & G. lamblia GPRT & Human \\
& & HGPRT \\
\hline GP3 & 52 & 86 \\
GP5 & 55 & 114 \\
GP13 & 72 & $>100\left(10.5^{c}\right)$ \\
\hline
\end{tabular}

${ }^{a}$ Compound identification.

${ }^{b} \mathrm{IC}_{50}, 50 \%$ inhibitory concentration.

${ }^{c}$ Remaining enzyme activity (\%) at the stated inhibitor concentration.

more selective scaffold that would occupy the purine binding portion of the active site. For example, a library of synthetically accessible carboxylic acids coupled to the $m$-aminophenol or $p$-chlorophenethylamine could be screened in silico to produce better alternatives to phthalimide in the context of the guanine-binding cleft of GPRT. The results of this investigation will be reported in due course.

In conclusion, we have used virtual combinatorial library screening across the representatives of the PRT gene family to make in silico predictions of combinatorial library performance against a panel of related enzymes. This approach has been applied to the discovery of micromolar inhibitors of $G$. lamblia GPRT. Phthalimide-based GPRT inhibitors displayed low to moderate selectivity for the parasite enzyme. Extension of the virtual screening matrix may prove useful in the process of scaffold selection for the purine binding site.

\section{ACKNOWLEDGMENTS}

We thank Paul Ortiz de Montellano for his support and insights in the course of this project. We are also grateful to Kenneth Foreman for helpful discussions.

This work was supported by the National Institutes of Health (grant AI-19319 to C.C.W. and grant GM31497 to I.D.K.).

\section{REFERENCES}

1. Adam, R. D. 1991. The biology of Giardia spp. Microbiol. Rev. 55:706-732.

2. Aronov, A. M., S. Suresh, F. S. Buckner, W. C. Van Voorhis, C. L. M. J. Verlinde, F. R. Opperdoes, W. G. J. Hol, and M. H. Gelb. 1999. Structurebased design of submicromolar, biologically active inhibitors of trypanosomatid glyceraldehyde-3-phosphate dehydrogenase. Proc. Natl. Acad. Sci. USA 96:4273-4278.

3. Aronov, A. M., N. R. Munagala, P. R. Ortiz de Montellano, I. D. Kuntz, and C. C. Wang. 2000. Rational design of novel antimicrobials: blocking purine salvage in a parasitic protozoan. Biochemistry 39:4684-4691.

4. Bressi, J. C., J. Choe, M. T. Hough, F. S. Buckner, W. C. Van Voorhis, C. L. M. J. Verlinde, W. G. J. Hol, and M. H. Gelb. 2000. Adenosine analogues as inhibitors of Trypanosoma brucei phosphoglycerate kinase: elucidation of a novel binding mode for a 2-amino- $N$-6-substituted adenosine. J. Med. Chem. 43:4135-4150.

5. Chin, M. S., and C. C. Wang. 1994. Isolation, sequencing and expression of the gene encoding hypoxanthine-guanine-xanthine phosphoribosyltransferase of Tritrichomonas foetus. Mol. Biochem. Parasitol. 63:221-229.

6. Chowdhury, S. F., V. B. Villamor, R. H. Guerrero, I. Leal, R. Brun, S. L. Croft, J. M. Goodman, L. Maes, L. M. Ruiz-Perez, D. G. Pacanowska, and I. H. Gilbert. 1999. Design, synthesis, and evaluation of inhibitors of trypanosomal and leishmanial dihydrofolate reductase. J. Med. Chem. 42:43004312.

7. Craig, S. P., III, and A. E. Eakin. 2000. Structure-based inhibitor design. Vitam. Horm. 58:149-169.

8. Eads, J. C., G. Scapin, Y. Xu, C. Grubmeyer, and J. C. Sacchettini. 1994. The crystal structure of human hypoxanthine-guanine phosphoribosyltransferase with bound GMP. Cell 78:325-334.

9. Ewing, T. J. A., and I. D. Kuntz. 1997. Critical evaluation of search algorithms for automated molecular docking and database screening. J. Comput. Chem. 18:1175-1189.

10. Focia, P. J., S. P. Craig, III, R. Nieves-Alicea, R. J. Fletterick, and A. E. 
Eakin. 1998. A $1.4 \AA$ crystal structure for the hypoxanthine phosphoribosyltransferase of Trypanosoma cruzi. Biochemistry 37:17120-17127.

11. Gutteridge, W. E. 1997. Designer drugs: pipe-dreams or realities? Parasitology 114:S145-S151.

12. Henriksen, A., N. Aghajari, K. F. Jensen, and M. Gajhede. 1996. A flexible loop at the dimer interface is a part of the active site of the adjacent monomer of Escherichia coli orotate phosphoribosyltransferase. Biochemistry 35:3803-3809.

13. Heroux, A., E. L. White, L. J. Ross, and D. W. Borhani. 1999. Crystal structures of the Toxoplasma gondii hypoxanthine-guanine phosphoribosyltransferase-GMP and -IMP complexes: comparison of purine binding interactions with the XMP complex. Biochemistry 38:14485-14494.

14. Kanaaneh, J., S. P. Craig III, and C. C. Wang. 1994. Differential inhibitory effects of GMP-2',3'-dialdehyde on human and schistosomal hypoxanthineguanine phosphoribosyltransferases. Eur. J. Biochem. 223:595-601.

15. Khan, M. O. F., S. E. Austin, C. Chan, H. Yin, D. Marks, S. N. Vaghjiani, H. Kendrick, V. Yardley, S. L. Croft, and K. T. Douglas. 2000. Use of an additional hydrophobic binding site, the $\mathrm{Z}$ site, in the rational drug design of a new class of stronger trypanothione reductase inhibitor, quaternary alkylammonium phenothiazines. J. Med. Chem. 43:3148-3156.

16. Li, R., X. Chen, B. Gong, P. M. Selzer, Z. Li, E. Davidson, G. Kurzban, R. E. Miller, E. O. Nuzum, J. H. McKerrow, R. J. Fletterick, S. A. Gillmor, C. S. Craik, I. D. Kuntz, F. E. Cohen, and G. L. Kenyon. 1996. Structure-based design of parasitic protease inhibitors. Bioorg. Med. Chem. 4:1421-1427.

17. Lipinski, C. A., F. Lombardo, B. W. Dominy, and P. J. Feeney. 1997. Experimental and computational approaches to estimate solubility and permeability in drug discovery and development settings. Adv. Drug Delivery Rev. 23:3-25.

18. Queen, S. A., D. Vander Jagt, and P. Reyes. 1988. Properties and substrate specificity of a purine phosphoribosyltransferase from the human malaria parasite, Plasmodium falciparum. Mol. Biochem. Parasitol. 2:123-133.

19. Ring, C. S., E. Sun, J. H. McKerrow, G. K. Lee, P. J. Rosenthal, I. D. Kuntz, and F. E. Cohen. 1993. Structure-based inhibitor design by using protein models for the development of antiparasitic agents. Proc. Natl. Acad. Sci. USA 90:3583-3587.

20. Scapin, G., D. H. Ozturk, C. Grubmeyer, and J. C. Sacchettini. 1995. The crystal structure of the orotate phosphoribosyltransferase complexed with orotate and $\alpha$-D-5-phosphoribosyl-1-pyrophosphate. Biochemistry 34:1074410754.

21. Schumacher, M. A., D. Carter, D. S. Roos, B. Ullman, and R. G. Brennan.
1996. Crystal structures of Toxoplasma gondii HGXPRTase reveal the catalytic role of a long flexible loop. Nat. Struct. Biol. 3:881-887.

22. Shi, W., C. M. Li, P. C. Tyler, R. H. Furneaux, S. M. Cahill, M. E. Girvin, C. Grubmeyer, V. L. Schramm, and S. C. Almo. 1999. The $2.0 \AA$ structure of malarial purine phosphoribosyltransferase in complex with a transition-state analogue inhibitor. Biochemistry 38:9872-9880.

23. Shi, W., N. R. Munagala, C. C. Wang, C. M. Li, P. C. Tyler, R. H. Furneaux, C. Grubmeyer, V. L. Schramm, and S. C. Almo. 2000. Crystal structures of Giardia lamblia guanine phosphoribosyltransferase at $1.75 \AA$. Biochemistry 39:6781-6790.

24. Shi, W., C. M. Li, P. C. Tyler, R. H. Furneaux, C. Grubmeyer, V. L. Schramm, and S. C. Almo. 1999. The 2.0 angstrom structure of human hypoxanthineguanine phosphoribosyltransferase in complex with a transition-state analog inhibitor. Nat. Struct. Biol. 6:588-593.

25. Skillman, A. G. 2000. Ph.D. thesis. University of California, San Francisco.

26. Sommer, J. M., H. Ma, and C. C. Wang. 1996. Cloning, expression and characterization of an unusual guanine phosphoribosyltransferase from $G i$ ardia lamblia. Mol. Biochem. Parasitol. 78:185-193.

27. Somoza, J. R., A. G. Skillman, Jr., N. R. Munagala, C. M. Oshiro, R. M. A. Knegtel, S. Mpoke, R. J. Fletterick, I. D. Kuntz, and C. C. Wang. 1998. Rational design of novel antimicrobials: blocking purine salvage in a parasitic protozoan. Biochemistry 37:5344-5348.

28. Somoza, J. R., M. S. Chin, P. J. Focia, C. C. Wang, and R. J. Fletterick. 1996. Crystal structure of the hypoxanthine-guanine-xanthine phosphoribosyltransferase from the protozoan parasite Tritrichomonas foetus. Biochemistry 35:7032-7040.

29. Vos, S., R. J. Parry, M. R. Burns, J. De Jersey, and J. L. Martin. 1998. Structures of free and complexed forms of Escherichia coli xanthine-guanine phosphoribosyltransferase. J. Mol. Biol. 282:875-889.

30. Walters, W. P., M. T. Stahl, and M. A. Murcko. 1998. Virtual screening-an overview. Drug Discov. Today 3:160-178.

31. Wang, C. C. 1984. Parasite enzymes as potential targets for antiparasitic chemotherapy. J. Med. Chem. 27:1-9.

32. Wang, C. C. 1997 . Validating targets for antiparasite chemotherapy. Parasitology 114:S31-S44

33. Wang, C. C., and S. Aldritt. 1983. Purine salvage networks in Giardia lamblia. J. Exp. Med. 158:1703-1712.

34. Yuan, L., S. P. Craig, J. H. McKerrow, and C. C. Wang. 1992. Steady-state kinetics of the schistosomal hypoxanthine-guanine phosphoribosyltransferase. Biochemistry 31:806-810. 